\title{
МЕТОД РАСЧЕТА КОНЦЕНТРАЦИОННЫХ И ТЕМПЕРАТУРНОГО ПРОФИЛЕЙ ПРОТИВОТОЧНОГО ДВУХФАЗНОГО ЖИДКОСТНОГО РЕАКТОРА
}

\author{
(Представил О. Эйзен)
}

Общие теоретические аспекты математического моделирования и расчета противоточных реакторов колонного типа для проведения газожидкостных реакций рассмотрены многими авторами, в т. ч. авторами широко известных монографий Дж. Астарита, П. В. Данквертса, А. С. Железняка и И. И. Иоффе $\left[{ }^{1-3}\right]$. В то же время в литературе пока мало внимания уделялось вычислительным аспектам решения этих задач, хотя и отмечались большие трудности в их решении.

Математическая модель двухфазного неизотермического реактора состоит из системы дифференциальных уравнений материальных и теплового балансов. Для такой системы уравнений, как правило, невозможно предложить аналитическое решение и поэтому ее решают методом численного интегрирования. Поскольку в случае противоточного реактора начальные условия концентраций компонентов и температуры задаются для обеих фаз на разных концах аппарата, то расчет его, в том числе определение концентрационных и температурных профилей, связан с решением двуточечной краевой задачи. Для решения такой задачи можно предложить разные итерационные методы, например, метод Ньютона, а также методы квазилинеаризации, Вольфа и др. В данном случае при использовании всех этих методов возникают трудности, связанные с несходимостью, чувствительностью к начальному приближению, значительным числом итерации, потребностью в большой машинной памяти и т. д. Практика применения коллокационного метода пока ограничивается решением сравнительно несложных задач с малочисленными переменными.

Наличие значительных трудностей вычисления отмечается уже в случае решения довольно простых двуточечных краевых задач массопередачи, в том числе при расчете колонн для неизотермической абсорбции. Для решения этих задач в последнее время предложены и релаксационные методы. Так, например, Дж. Р. Боурн, У. Стокар, Г. К. Коггэн и К. Р. Уйльке [4,5] для расчета абсорбционных колонн предлагают применять так называемый динамический метод, имитирующий пуск в действие реального аппарата. Аналогичен ему и метод, использованный Х. Хартигом [6], для расчета изотермического химического реактора в случае одного реагирующего и переходящего компонента. Принимая основную идею этого метода, мы приспособили его для расчета противоточных двухфазных неизотермических жидкостных реакторов, 
где одновременно со сложной химической реакцией протекает многокомпонентная разнонаправленная массопередача.

В качестве примера рассмотрим противоточный реактор типа насадочной колонны, где сверху вниз стекает жидкая фаза - жидкий реагент вместе с растворенным газовым реагентом и продуктами реакции, а снизу вверх движется газовая фаза-газовый реагент вместе с пара́ми компонентов жидкости. В колонне происходит многокомпонентный разнонаправленный массообмен-абсорбция газового реагента в жидкости с одновременным испарением летучих компонентов. Одновременно с массопередачей протекает сложная жидкофазная химическая реакция. Неизотермичность процесса вызывается тепловым эффектом реакции, теплотой парообразования компонентов и теплопередачей в окружающую среду (к теплоносителю). Объемная скорость газовой фазы изменяется вдоль колонны, объемную скорость жидкости можно с некоторым приближением считать постоянной.

Если обе фазы движутся в режиме идеального вытеснения, математическая модель реактора выражается в обобщенном виде следующей системой уравнений:

$$
\begin{aligned}
& V_{\text {ж }} \frac{d c_{i}}{d h}= \pm R+N_{i}, \\
& \frac{d\left(V_{\mathrm{r}} y_{i}\right)}{d h}=\frac{d g_{i}}{d h}=N_{i}, \\
& V_{\text {ж }} \sum_{i=1}^{n}\left(C_{p_{t}} c_{i}\right) \frac{d t}{d h}=-R \Delta q_{p}+\sum_{i=1}^{n} N_{i} \Delta q_{\Pi i}+Q \text {, }
\end{aligned}
$$

где $V_{\text {ж и }} V_{\text {г }}$ - объемные скорости жидкой и газовой фаз; $c_{i}$ и $y_{i}$ концентрации $i$-го компонента в жидкой и газовой фазах; $t-$ температура; $h$ - текущая высота реактора; $C_{p i}-$ теплоемкость $i$-го компонента; $\Delta q_{p}$ - тепловой эффект реакции; $\Delta q_{п i}$ - теплота фазового превращения (абсорбции, испарения) $i$-го компонента; $N_{i}-$ скорость межфазной массопередачи $i$-го компонента в направлении от жидкости к газу; $Q-$ скорость теплопередачи в окружающую среду (к теплоносителю); $R$ - скорость химической реакции (с положительным знаком в первом уравнении для компонентов, расходуемых в ходе реакции). Скорости всех вышеприведенных потоков $\left(N_{i}, Q\right.$ и $\left.R\right)$ отнесены к единице высоты реактора и могут быть выражены соответствующими уравнениями массо-, теплопередачи и химической кинетики, т. е. являются функциями входящих в уравнения $(1-3)$ переменных: $N_{i}(\bar{c}, \bar{g}, t), Q\left(t, t_{c}\right)$ и $R(\bar{c}, t)$. При этом $\bar{c}-$ концентрации компонентов жидкой фазы; $\bar{g}$ - газовые потоки по компонентам; $t_{c}$ - температура окружающей среды.

Краевые условия:

и

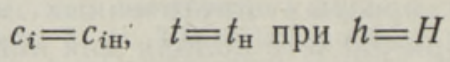

$$
g_{i}=g_{i \text { н }} \text { при } h=0 .
$$

Для расчета концентрационных и температурного профилей делим высоту реактора (колонны) $H$ на $k$ слайдов высотами $\Delta h=\frac{H}{k}$.

Сначала «пускаем в колонну газ», т. е. присваиваем потокам газовых компонентов в каждом $j=1, \ldots, k$ слайде (рис. 1) исходное значение $g_{i j}$. Эти значения могут быть равными начальным условиям 


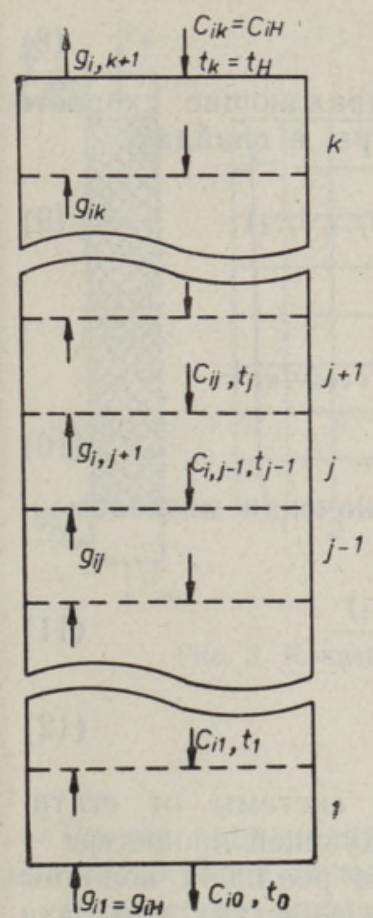

Рис. 1. Разделение реактора на слайды.

Рис. 2. Блок-схема расчета.

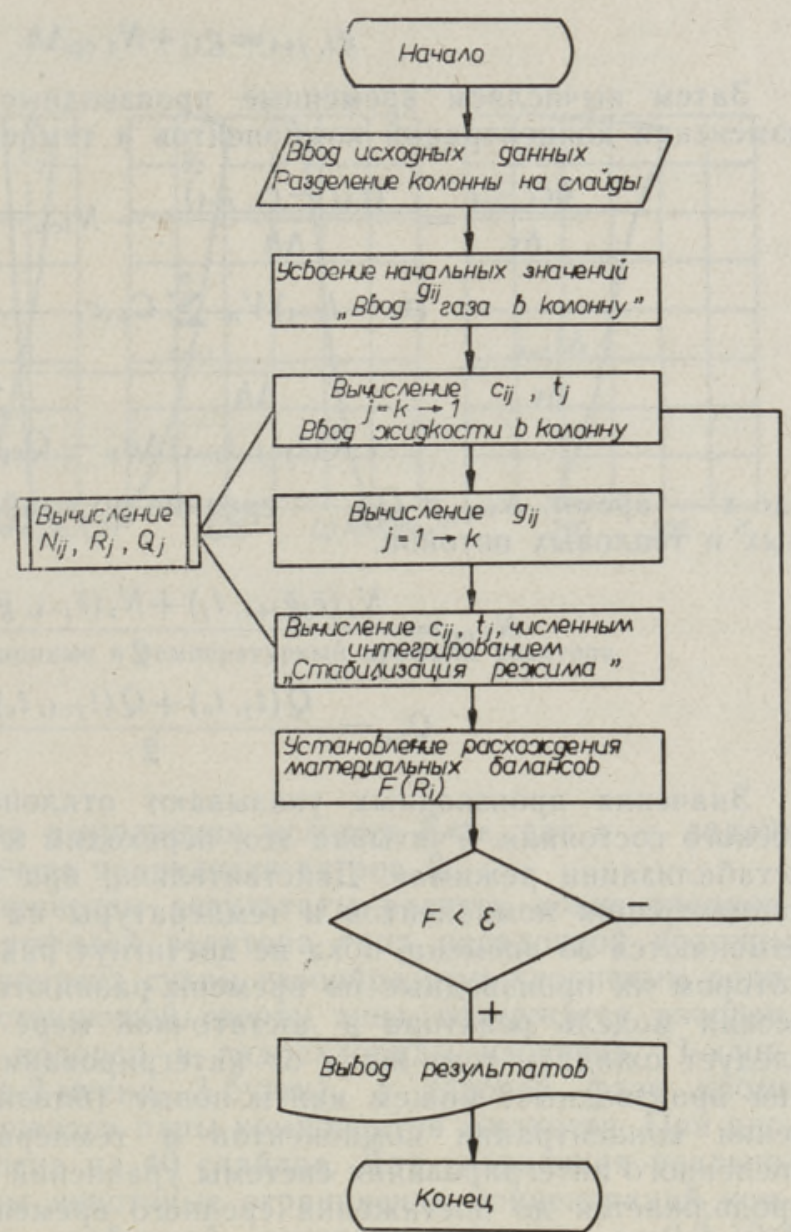

$g_{i j}=g_{i н}$, но с целью ускорения сходимости решения можем принять исходный концентрационный профиль по возможности близким к ожидаемому.

Затем «пускаем в колонну жидкость», т. е. вычисляем концентрацию компонентов и температуру жидкости во всех слайдах последовательно от верхнего слайда к нижнему. Для расчета используем конечно-разностные уравнения материальных и тепловых балансов, полученные из приведенных выше дифференциальных уравнений (1) и (3).

$$
\begin{gathered}
c_{i, j-1}=c_{i j}-\frac{\left[N_{i}\left(\bar{c}_{j}, \bar{g}_{j}, t_{j}\right) \pm R\left(\bar{c}_{j}, t_{j}\right)\right] \Delta h}{V_{\text {ж }}}, \\
t_{j-1}=t_{j}-\frac{\left[\sum_{i=1}^{n} N_{i}\left(\bar{c}_{j}, \bar{g}_{j}, t_{j}\right) \Delta q_{\Pi i}-R\left(\bar{c}_{j}, t_{j}\right)+Q\left(t_{j}, t_{c}\right)\right] \Delta h}{V_{\text {ж }} \sum_{i=1}^{n} C_{p_{t}} c_{i j}} .
\end{gathered}
$$

Далее проводим расчет газовых потоков по конечно-разностным уравнениям, полученным из дифференциального уравнения (2). Расчет проводим в направлении снизу вверх. 


$$
g_{i, j+1}=g_{i j}+N_{i \mathrm{cp}} \Delta h .
$$

Затем вычисляем временные производные, выражающие скорость изменения концентраций компонентов и температуры в слайдах,

$$
\begin{gathered}
\frac{\delta c_{i, j-1}}{\delta \tau}=\frac{\left(c_{i j}-c_{i, j-1}\right)}{\Delta h}-N_{i \mathrm{cp}}-R\left(\bar{c}_{j-1}, \dot{t}_{j-1}\right), \\
\frac{\delta t_{j-1}}{\delta \tau}=\frac{\left(t_{j}-t_{j-1}\right) V_{\text {世 }} \sum_{i=1}^{n} C_{p i} c_{i}}{\Delta h}-\sum_{i=1}^{n} N_{i \mathrm{cp}} \Delta q_{\Pi i}+ \\
+R\left(\bar{c}_{j-1}, t_{j-1}\right) \Delta q_{p}-Q_{\mathrm{cp}}
\end{gathered}
$$

где $\tau-$ время; $N_{i c p}$ и $Q_{\text {cp }}-$ средние по слайду значения массообменных и тепловых потоков.

$$
\begin{gathered}
N_{i \mathrm{cp}}=\frac{N_{i}\left(\bar{c}_{j} \bar{g}_{j+1}, t_{j}\right)+N_{i}\left(\bar{c}_{j-1}, \bar{g}_{j}, t_{j-1}\right)}{2}, \\
Q_{\mathrm{cp}}=\frac{Q\left(t_{j}, t_{c}\right)+Q\left(t_{j-1}, t_{c}\right)}{2} .
\end{gathered}
$$

Значения производных указывают отклонение системы от статического состояния. Учитывая это, переходим к следующей процедуре «стабилизации режима». Действительно, при пуске реальной колонны концентрация компонентов и температуры на всех участках аппарата изменяются во времени, пока не достигнут равновесного состояния, при котором их производные по времени равняются нулю. Если математическая модель реактора в достаточной мере отвечает реальности, то следует ожидать того же и от интегрирования. Зная числовые значения производных, можем найти новые (близкие к равновесным) значения концентрации компонентов и температуры в слайдах путем численного интегрирования системы уравнений $(9-10)$. Интегрирование продолжается до достижения среднего времени пребывания жидкости в слайде

$$
\tau_{c}=\frac{H_{u} \pi D^{2} \Delta h}{4 V_{\text {ж }}},
$$

где $D$ - диаметр колонны, $H_{u}-$ удерживающая способность насадки по жидкости. Для решения рассматриваемой задачи оказалось достаточным применение метода первого порядка Рунге-Кутта и разделение всего промежутка интегрирования лишь на 3 шага.

Далее снова следует вычисление концентрации компонентов жидкости и температуры по уравнениям (6) и (7), расчет газовых потоков по уравнению (8) и новое интегрирование уравнения (9) и (10). Весь цикл повторяется до максимального совпадения всех покомпонентных материальных балансов по всей колонне. В рассматриваемом случае количество прореагировавшего вещества можем выразить через материальные балансы отдельных компонентов следующим образом:

$$
R_{i}= \pm\left(g_{i, k+1}-g_{i 1}\right) \pm V_{\text {ж }}\left(c_{i 0}-c_{i k}\right),
$$

а критерий расхождения материальных балансов - формулой:

$$
F=\sum_{i=1}^{n}\left\{R_{i}-\frac{\sum_{i=1}^{n} R_{i}}{n}\right\rfloor^{2}
$$



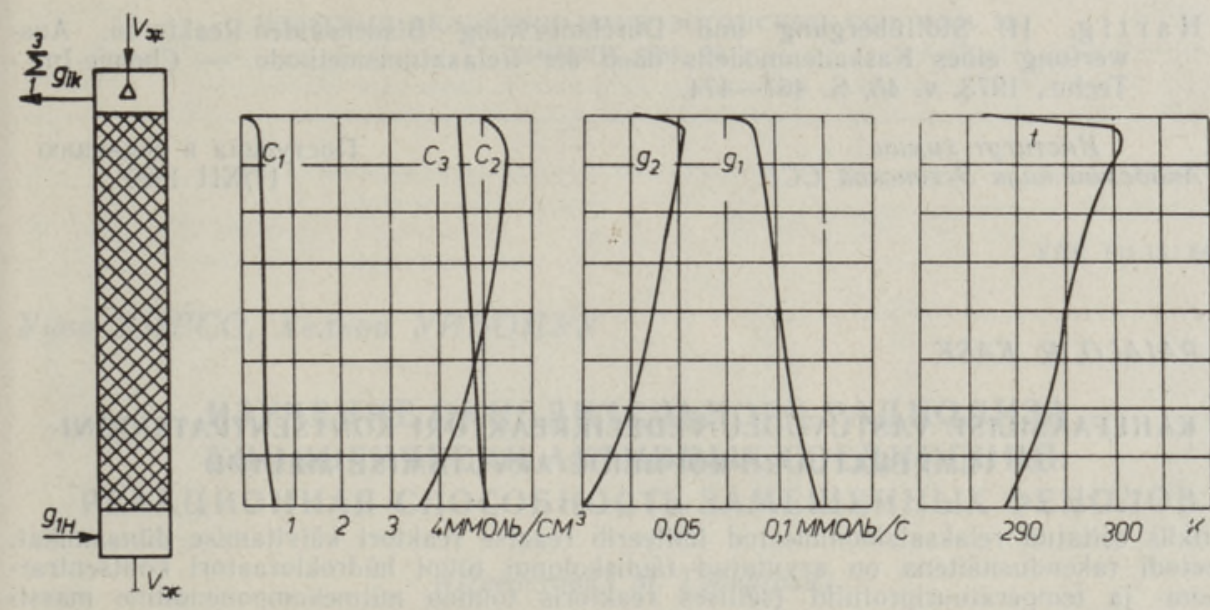

Рис. 3. Концентрационные и температурный профили реактора.

Расчет производится до выполнения условия $F<\varepsilon$, где $\varepsilon-$ заданное число. Блок-схема расчета приводится на рис. 2.

В качестве примера приведем результаты расчета концентрационного и температурного профилей реактора типа насадочной колонны для гидрохлорирования изопрена сухим газообразным хлористым водородом. В жидкой фазе, стекающей сверху вниз, содержатся изопрен, растворенный хлористый водород и гидрохлориды изопрена (1-хлор3-метил-2-бутен и 2-хлор-2-метил-3-бутен), в газовой фазе кроме хлористого водорода содержатся пары компонентов жидкости. При расчете колонна была разделена на 40 слайдов. Для сохранения реальности решения были введены некоторые ограничения концентраций компонентов. Для получения требуемой точности решения $\varepsilon=0,2 \cdot 10^{-5}$ понадобились 3 итерации. Расчет проводился на ЭВМ ЕС-1022. Время вычислений - 2 мин. Рассчитанные концентрационные и температурный профили приводятся на рис. 3.

Следует отметить, что предлагаемую нами методику можно применять при расчете любого противоточного двухфазного химического реактора, хотя рассмотренная выше задача - одна из самых сложных. При изотермичности процесса, ограничении числа компонентов, отсутствии испарения и постоянстве газового потока задача в значительной мере упрощается. В случае мгновенной реакции можно применять указанные методики расчета абсорбционных колонн.

\section{Л ИТЕРАТ УРА}

1. А с т а ри т а Дж. Массопередача с химической реакцией. Л., 1971.

2. Д а н к в е р тс П. В. Газо-жидкостные реакции. М., 1973.

3. Железняк А. С., Иоффе И. И. Методы расчета многофазных жидкостных реакторов. Л., 1974.

4. Bourne, J. R., Stockar, U., Coggan, G. C. Gas absorption with heat effects. I. A new computational method. Ind. Eng. Chem. Process Des. Dev., 1974 , v. $13, \mathrm{~N} 2$, p. $115-123$.

5. Stockar, U., Wilke, C. R. Rigorous and shortcut design calculations for gas absorption involving large heat effects. 1. A new computational method for packed gas absorbers. Ind. Eng. Chem. Fundam., 1977, v. 16, N 1, p. 88-93. 
6. Hartig, H. Stoffübergang und Durchmischung Blasensäulen-Reaktoren. Auswertung eines Kaskadenmodells nach der Relaxationsmethode. - Chemie-Ing.Techn., 1973 , v. 45 , S. $467-474$.

Институт химии

Академии наук Эстонской ССР

Поступила в редакцию 17/XII 1980

G. RAJALO, R. KASK

\section{KAHEFAASILISE VASTUVOOLU-VEDELIKREAKTORI KONTSENTRATSIOONI- JA TEMPERATUURIPROFIILIDE ARVUTAMISE MEETOD}

Artiklis esitatud relaksatsioonimeetod imiteerib reaalse reaktori käivitamise dünaamikat. Meetodi rakendusnäitena on arvutatud täidiskolonni tüüpi hüdrokloraatori kontsentratsiooni- ja temperatuuriprofiilid (sellises reaktoris toimub mitmekomponendiline massiülekanne koos keemilise liitreaktsiooniga).

G. RAJALO, R. KASK

\section{A METHOD FOR CALCULATING CONCENTRATION AND TEMPERATURE PROFILES IN A COUNTERCURRENT TWO-PHASE LIQUID REACTOR}

A relaxation method for calculating a countercurrent two-phase liquid reactor simulating the dynamics of the start-up procedure of the real column is presented. An example is given for applying the method to the calculation of concentration and temperature profiles for a packed-bed hydrochlorator in which simultaneous multicomponent mass transfer and complex chemical reactions take place. 